\title{
TWENTY YEARS OF TEVATRON OPERATION
}

\author{
J. C. Theilacker \\ Fermi National Accelerator Laboratory \\ Batavia, IL, 60510, USA
}

\begin{abstract}
The superconducting Tevatron accelerator at Fermi National Accelerator Laboratory (Fermilab) has surpassed twenty years of operation. The Tevatron is still the highest energy particle accelerator in the world and will remain so until the commissioning of the LHC in Europe later this decade. The Tevatron has operated in a Fixed Target mode, accelerating a proton beam into stationary targets/detectors, as well as a Colliding Beam mode, continuously colliding counter rotating beams of protons and antiprotons. Upon completion, the Tevatron cryogenic system became the world's largest helium refrigeration system. In 1993, the Tevatron cryogenic system was given the designation of International Historic Mechanical Engineering Landmark by the American Society of Mechanical Engineers. The operational history, experiences and statistics of the Tevatron, with an emphasis on the cryogenic system, is presented. Improvements, upgrades and current challenges of the cryogenic system are discussed.
\end{abstract}

\section{DESCRIPTION}

The Tevatron cryogenic system is a hybrid system consisting of a large central helium liquefier and twenty-four satellite refrigerators [1-2]. This geometry was driven mainly by the choice of warm iron superconducting magnets. The choice of warm iron magnet design was mainly driven by space constraints in the existing Main Ring accelerator tunnel. The Tevatron was a higher energy accelerator stage which was required to fit below the Main Ring and through existing voids in the Main Ring magnet stands.

It is desirable to locate the iron as close to the superconducting coils as practical, in order to maximize the central magnetic field. This results in short thermal standoffs between $4 \mathrm{~K}$ and $300 \mathrm{~K}$. The flexibility of a small diameter collared coil assembly also requires many supports to ensure uniform central magnetic field. As a result, a warm iron magnet design inherently has a high heat leak to $4 \mathrm{~K}$. 
The high heat load associated with warm iron magnets necessitated distributing refrigeration over short distances. This resulted in a hybrid cryogenic system design consisting of a large central helium liquefier (CHL) and twenty-four $1 \mathrm{~kW}$ capacity satellite refrigerators. At the time of the Tevatron design and construction, $1 \mathrm{~kW}$ class helium refrigerators required reciprocating expansion engines. Reciprocating expanders, as opposed to turbo expanders, had reliability and maintenance issues which needed to be addressed [3].

It was originally envisioned to truck liquid helium from the CHL to the twenty-four satellite refrigerators in 500 liter dewars. Fortunately, this plan was abandoned with the design and construction of a thermally efficient $7 \frac{1 / 4}{4 m}$ liquid helium-liquid nitrogen transfer line [4].

The magnets were designed to be cooled by a two-phase helium flow which heat exchanged to subcooled liquid that flowed through the superconducting coils. Tests performed in the 1980s showed that the two-phase flow regime was highly stratified, resulting in higher magnet coil temperatures than anticipated [5-6].

In order to operate the Tevatron at higher beam energies, a large cryogenic system upgrade was completed in the 1990s [7]. Cold helium vapor compressors were required to lower the operating temperature of the Tevatron in order to achieve the higher energies [8]

\section{OPERATIONAL HISTORY}

An operational timeline is shown in TABLE 1. The Tevatron was intended for Fixed Target as well as Colliding beam physics. During Fixed Target physics, protons are injected, ramped to full energy, slowly extracted to parallel experiments, the magnets are then ramped down and the cycle repeats. The cycling of the magnet energy means that the refrigeration system must satisfy both the static and dynamic heat loads. The dynamic heat load is predominately hysteresis within the superconductor. Injecting and extracting protons on each cycle (57 seconds) also increases the odds for stray beam to cause a quench.

During colliding beam physics, magnets are ramped to full energy with counter rotating beams of protons and antiprotons. Collisions typically are allowed to continue at full energy for a 24 hour period. This significantly reduces the cryogenic load by virtually eliminating the dynamic loss. The lack of dynamic losses in colliding beam physics mode allows the Tevatron to operate at higher energy than in fixed target physics.

The Tevatron has 24 satellite refrigerators, each cooling two $125 \mathrm{~m}$ long magnet strings. In order to work on or replace a cold component, the entire satellite refrigerator and $250 \mathrm{~m}$ of magnets are warmed to $300 \mathrm{~K}$.

Since its initial cooldown in 1983, there have been 262 house thermal cycles to $300 \mathrm{~K}$. This is equivalent to over ten full ring thermal cycles. Of the 262 house thermal cycles, 120 $(46 \%)$ are due to five full ring thermal cycles. There were $72(27 \%)$ unscheduled warmups, representing 61 events, that required immediate attention. The remaining 70 warm-ups $(27 \%)$ were scheduled. FIGURE 1 presents a distribution of the reasons for each warm-up.

With crews working around the clock, a repair typically takes between 5 and 7 days. This includes 2 days for warm-up, 1 day for cooldown and 2-4 days for repair and safety review. Cooldown from $300 \mathrm{~K}$ is with a $4.5 \mathrm{~K}$ liquid helium cooling wave. No cooldown rate constraints have been applied to the Tevatron. Typically, the length of the $4.5 \mathrm{~K}$ to 300 $\mathrm{K}$ cooling wave is on the order of one magnet $(6.4 \mathrm{~m})$. The cooldown wave remains abrupt through the entire magnet string. 
TABLE 1. Tevatron Operational History

\begin{tabular}{|c|c|c|}
\hline Mar 1983 & & Last Tevatron magnet installed \\
\hline Apr 1983 & May 1983 & E \& F sector tests (with beam) \\
\hline Jun 1983 & Sep 1983 & Accelerated and extracted $512 \mathrm{GeV}$ beam and Stored $700 \mathrm{GeV}$ beam \\
\hline Oct 1983 & Jan 1984 & $400 \mathrm{GeV}$ Fixed Target Physics Run \\
\hline Feb 1984 & & $800 \mathrm{GeV}$ beam extraction and storage tests \\
\hline Mar 1984 & Jun 1984 & $800 \mathrm{GeV}$ Fixed Target Physics Run \\
\hline Jul 1984 & Nov 1984 & Shutdown for dipole repair, D0 overpass and antiproton source construction \\
\hline Dec 1984 & & Accelerator startup \\
\hline Jan 1985 & Jun 1985 & $800 \mathrm{GeV}$ Fixed Target Physics Run \\
\hline Jul 1985 & Aug 1985 & $800 \mathrm{GeV}$ Physics Run and antiproton source commissioning \\
\hline Sep 1985 & Oct 1985 & Collider test run \\
\hline Nov 1985 & Jul 1986 & Shutdown for B0 overpass and D0 experimental hall construction \\
\hline Aug 1986 & Jan 1987 & Accelerator startup \\
\hline Feb 1987 & Apr 1987 & $900 \mathrm{GeV}$ Collider Physics Run \\
\hline May 1987 & & Switchover to Fixed Target Physics \\
\hline Jun 1987 & Feb 1988 & $800 \mathrm{GeV}$ Fixed Target Physics Run \\
\hline Mar 1988 & May 1988 & Shutdown for dipole repair, D0 overpass and B0 shielding \\
\hline Jun 1988 & & Accelerator startup \\
\hline Jul 1988 & May 1989 & $900 \mathrm{GeV}$ Collider Physics Run \\
\hline Jun 1989 & & Accelerator studies \\
\hline Jul 1989 & Nov 1989 & Shutdown for dipole repair \\
\hline Dec 1989 & Jan 1990 & Accelerator startup \\
\hline Feb 1990 & Aug 1990 & $800 \mathrm{GeV}$ Fixed Target Physics Run \\
\hline Sep 1990 & Jun 1991 & Low beta magnet installation, shielding assessment and accelerator startup \\
\hline Jul 1991 & Jan 1992 & $800 \mathrm{GeV}$ Fixed Target Physics Run \\
\hline Feb 1992 & Aug 1992 & Switchover to Collider Physics and accelerator startup \\
\hline Sep 1992 & May 1993 & 900 GeV Collider Physics Run IA \\
\hline Jun 1993 & Nov 1993 & Shutdown for Tevatron high energy upgrade and $400 \mathrm{MeV}$ Linac upgrade \\
\hline Dec 1993 & Feb 1996 & 900 GeV Collider Physics Run IB \\
\hline Mar 1996 & Jul 1996 & Switchover to Fixed Target Physics and accelerator startup \\
\hline Aug 1996 & Aug 1997 & $800 \mathrm{GeV}$ Fixed Target Physics Run \\
\hline Sep 1997 & Mar 1999 & Shutdown for Main Injector tie in and C0 collision hall construction \\
\hline Sep 1998 & May 1999 & Main Injector commissioning and accelerator startup \\
\hline Jun 1999 & Jan 2000 & $800 \mathrm{GeV}$ Fixed Target Physics Run \\
\hline Feb 2000 & Jun 2000 & Switchover to Collider Physics and accelerator startup \\
\hline Jul 2000 & Oct 2000 & $980 \mathrm{GeV}$ Engineering Run \\
\hline Nov 2000 & Feb 2001 & Detector roll in and accelerator startup \\
\hline Mar 2001 & Sep 2003 & $980 \mathrm{GeV}$ Collider Physics Run \\
\hline
\end{tabular}

\section{CRYOGENIC DOWNTIME}

Analysis of downtime for accelerators at Fermilab can be misleading due to overlapping downtime with other systems. If a system has a known problem, but is able to continue operation, it is not uncommon for the problem to be corrected during downtime caused by another system. However, both, or more, systems are charged with the downtime. This accounting system is helpful to keep track of all work performed on the system, but results in unrealistic total downtime figures due to the multiple counting.

Downtime associated with the cryogenic system is divided into two categories, CHL and satellite refrigerators (TCRYO). Also included in this paper is the downtime associated with magnet quenches (TQUEN), since it is particularly disruptive to the cryogenic system. Downtime for CHL, TCRYO and TQUEN are given in TABLES 2-4, respectively, for all physics runs that have occurred since the commissioning of the Tevatron. 


\section{Tevatron House Warm-Up Analysis}

$1983-2003$

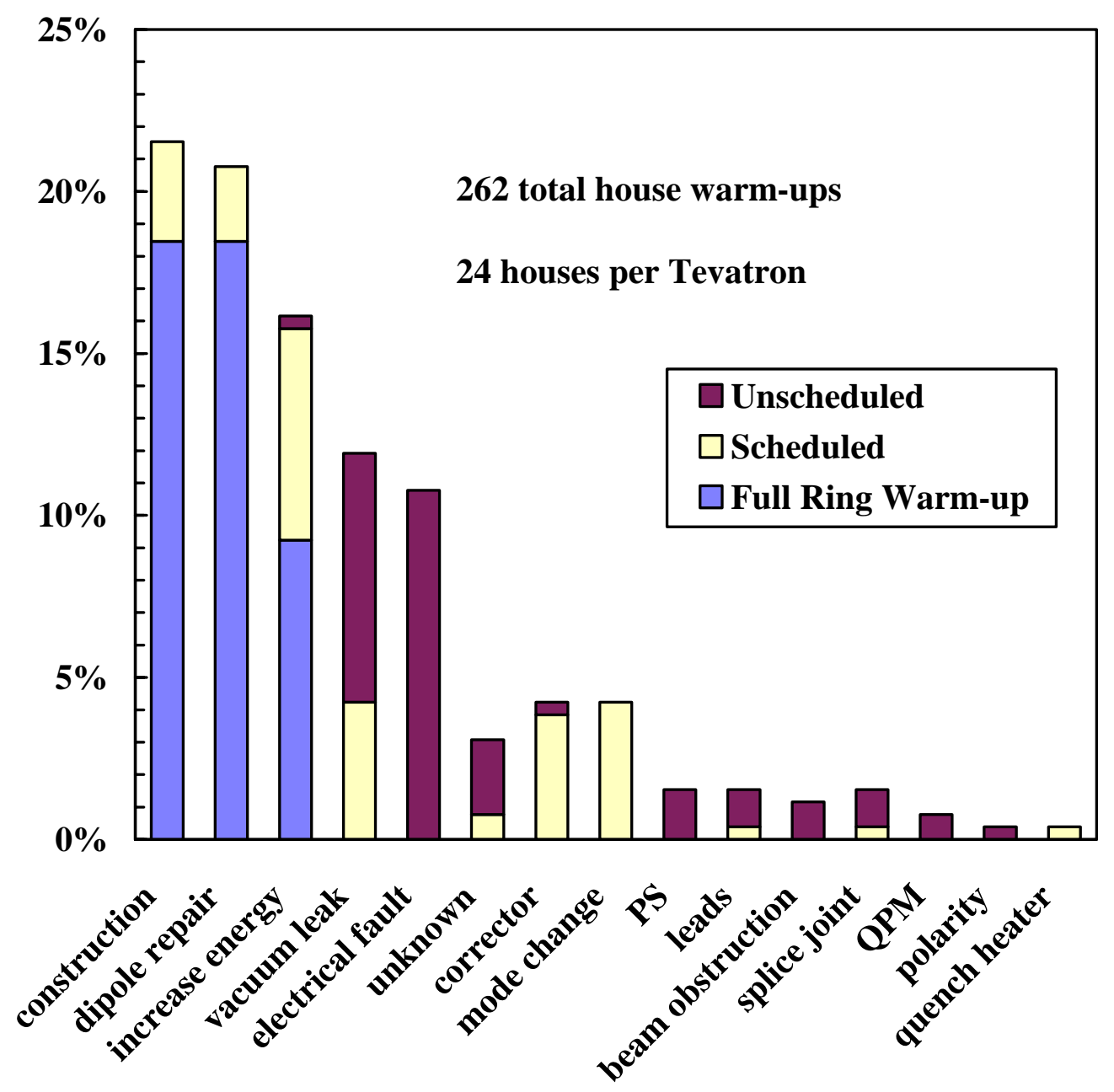

FIGURE 1. Reasons for Tevatron Magnet String Warm-ups

TABLE 2 shows that the downtime associated with the CHL was higher and with significantly more events, in early physics runs. Four major improvements were made to improve downtime.

1) Residual aluminum oxide dust from the original brazing of the plate-fin heat exchangers was blown out. It had been repeatedly plugging the turbine inlet filters.

2) A redundant third compressor was commissioned.

3) A liquid helium pump was commissioned which allows pumped liquid helium from storage dewars to be added to the plant production during times of high satellite refrigerator demand.

4) The development of a spectrographic nitrogen detector, which could reliably measure down to 1 PPM [9]. This led to better purification techniques and maintenance procedures. 
TABLE 2. Central Helium Liquefier Downtime Versus Physics Run

\begin{tabular}{|l|c|c|c|c|c|}
\hline Physics Run & $\begin{array}{c}\text { CHL } \\
\text { Downtime }\end{array}$ & $\begin{array}{c}\text { Run } \\
\text { Length }\end{array}$ & $\begin{array}{c}\# \\
\text { Events }\end{array}$ & $\begin{array}{c}\text { CHL } \\
\text { Downtime }\end{array}$ & $\begin{array}{c}\text { CHL } \\
\text { Availability }\end{array}$ \\
\hline [hours] & [hours] & {$[-]$} & {$[\%]$} & {$[\%]$} \\
\hline 980 GeV Collider Run II (to date) & 54.1 & 21,991 & 5 & $0.2 \%$ & $99.8 \%$ \\
\hline 980 GeV Collider Engr. Run (2000) & 30.2 & 2,736 & 1 & $1.1 \%$ & $98.9 \%$ \\
\hline 800 GeV Fixed Target (1999) & 35.4 & 5,376 & 2 & $0.7 \%$ & $99.3 \%$ \\
\hline 800 GeV Fixed Target (1996/7) & 13.7 & 9,600 & 2 & $0.1 \%$ & $99.9 \%$ \\
\hline 900 GeV Collider Run IB (1994/5) & 29.5 & 19,104 & 2 & $0.2 \%$ & $99.8 \%$ \\
\hline 900 GeV Collider Run IA (1992/3) & 32.3 & 6,528 & 3 & $0.5 \%$ & $99.5 \%$ \\
\hline 800 GeV Fixed Target (1991/2) & 3.3 & 4,225 & 10 & $0.1 \%$ & $99.9 \%$ \\
\hline 800 GeV Fixed Target (1990) & 4.1 & 4,703 & 1 & $0.1 \%$ & $99.9 \%$ \\
\hline 900 GeV Collider (1988/89) & 12.8 & 8,280 & 4 & $0.2 \%$ & $99.8 \%$ \\
\hline 800 GeV Fixed Target (1987/8) & 7.4 & 5,881 & 13 & $0.1 \%$ & $99.9 \%$ \\
\hline 900 GeV Collider (1987) & 0.0 & 2,351 & 0 & $0.0 \%$ & $100.0 \%$ \\
\hline 800 GeV Collider (1985) & 0.0 & 744 & 0 & $0.0 \%$ & $100.0 \%$ \\
\hline 800 GeV Fixed Target (1985) & 62.1 & 5,447 & 18 & $1.1 \%$ & $98.9 \%$ \\
\hline 800 GeV Fixed Target (1984) & 35.3 & 3,023 & 13 & $1.2 \%$ & $98.8 \%$ \\
\hline 400 GeV Fixed Target (1983/4) & 60.2 & 3,433 & 12 & $1.8 \%$ & $98.2 \%$ \\
\hline
\end{tabular}

TABLE 3 shows the downtime associated with the Tevatron satellite refrigeration system. The downtime for this system is higher than for CHL, as expected, due to the high number of systems (24) and the use of reciprocating expansion engines. It is interesting that the downtime is not consistently correlated with physics type (Fixed Target versus Collider). With the constant magnet ramping and the greater beam manipulation, one would expect Fixed Target physics to place a greater burden on the cryogenic system reliability.

Satellite refrigerator downtime was higher during early physics runs of the Tevatron. Two major improvements were made to improve downtime.

1) Improvements in alignment and materials used in our reciprocating expanders have improved the mean time between failures [3]. Operating times of one year are now common, allowing maintenance to take place during annual shutdowns.

2) Parts used to clamp conductor within dipole magnets were coming loose and becoming wedged within magnet quench relief valves. Parts were secured during 1984 and 1989 dipole repair shutdowns.

TABLE 3. Tevatron Satellite Refrigerator Downtime Versus Physics Run

\begin{tabular}{|l|c|c|c|c|c|}
\hline Physics Run & $\begin{array}{c}\text { TCRYO } \\
\text { Downtime }\end{array}$ & $\begin{array}{c}\text { Run } \\
\text { Length }\end{array}$ & $\begin{array}{c}\# \\
\text { Events }\end{array}$ & $\begin{array}{c}\text { TCRYO } \\
\text { Downtime }\end{array}$ & $\begin{array}{c}\text { TCRYO } \\
\text { Availability }\end{array}$ \\
\hline [hours] & [hours] & {$[-]$} & {$[\%]$} & {$[\%]$} \\
\hline 980 GeV Collider Run II (to date) & 282.1 & 21,991 & 95 & $1.3 \%$ & $98.7 \%$ \\
\hline 980 GeV Collider Engr. Run (2000) & 88.8 & 2,736 & 24 & $3.2 \%$ & $96.8 \%$ \\
\hline 800 GeV Fixed Target (1999) & 163.6 & 5,376 & 97 & $3.0 \%$ & $97.0 \%$ \\
\hline 800 GeV Fixed Target (1996/7) & 78.7 & 9,600 & 191 & $0.8 \%$ & $99.2 \%$ \\
\hline 900 GeV Collider Run IB (1994/5) & 190.6 & 19,104 & 166 & $1.0 \%$ & $99.0 \%$ \\
\hline 900 GeV Collider Run IA (1992/3) & 61.9 & 6,528 & 47 & $0.9 \%$ & $99.1 \%$ \\
\hline 800 GeV Fixed Target (1991/2) & 44.9 & 4,225 & 100 & $1.1 \%$ & $98.9 \%$ \\
\hline 800 GeV Fixed Target (1990) & 45.5 & 4,703 & 97 & $1.0 \%$ & $99.0 \%$ \\
\hline 900 GeV Collider (1988/89) & 74.5 & 8,280 & 47 & $0.9 \%$ & $99.1 \%$ \\
\hline 800 GeV Fixed Target (1987/8) & 77.8 & 5,881 & 136 & $1.3 \%$ & $98.7 \%$ \\
\hline 900 GeV Collider (1987) & 30.4 & 2,351 & 16 & $1.3 \%$ & $98.7 \%$ \\
\hline 800 GeV Collider (1985) & 7.0 & 744 & 8 & $0.9 \%$ & $99.1 \%$ \\
\hline 800 GeV Fixed Target (1985) & 118.5 & 5,447 & 280 & $2.2 \%$ & $97.8 \%$ \\
\hline 800 GeV Fixed Target (1984) & 187.6 & 3,023 & 420 & $6.2 \%$ & $93.8 \%$ \\
\hline 400 GeV Fixed Target (1983/4) & 204.3 & 3,433 & 408 & $6.0 \%$ & $94.0 \%$ \\
\hline
\end{tabular}


Table 4. Tevatron Magnet Quench Downtime Versus Physics Run

\begin{tabular}{|l|r|r|r|c|c|}
\hline Physics Run & $\begin{array}{c}\text { TQUEN } \\
\text { Downtime }\end{array}$ & $\begin{array}{c}\text { Run } \\
\text { Length }\end{array}$ & $\begin{array}{c}\# \\
\text { Events }\end{array}$ & $\begin{array}{c}\text { TQUEN } \\
\text { Downtime }\end{array}$ & $\begin{array}{c}\text { TQUEN } \\
\text { Availability }\end{array}$ \\
\hline 980 GeV Collider Run II (to date) & {$[$ hours] } & \multicolumn{1}{|c|}{$[$ hours] } & {$[-]$} & {$[\%]$} & {$[\%]$} \\
\hline 980 GeV Collider Engr. Run (2000) & 79.8 & 21,991 & 315 & $3.7 \%$ & $96.3 \%$ \\
\hline 800 GeV Fixed Target (1999) & 99.6 & 5,736 & 41 & $2.9 \%$ & $97.1 \%$ \\
\hline 800 GeV Fixed Target (1996/7) & 208.8 & 9,600 & 312 & $1.9 \%$ & $98.1 \%$ \\
\hline 900 GeV Collider Run IB (1994/5) & 594.5 & 19,104 & 247 & $3.1 \%$ & $96.9 \%$ \\
\hline 900 GeV Collider Run IA (1992/3) & 123.3 & 6,528 & 56 & $1.9 \%$ & $98.1 \%$ \\
\hline 800 GeV Fixed Target (1991/2) & 70.1 & 4,225 & 89 & $1.7 \%$ & $98.3 \%$ \\
\hline 800 GeV Fixed Target (1990) & 110.7 & 4,703 & 121 & $2.4 \%$ & $97.6 \%$ \\
\hline 900 GeV Collider (1988/89) & 194.5 & 8,280 & 112 & $2.3 \%$ & $97.7 \%$ \\
\hline 800 GeV Fixed Target (1987/8) & 211.5 & 5,881 & 215 & $3.6 \%$ & $96.4 \%$ \\
\hline 900 GeV Collider (1987) & 60.5 & 2,351 & 45 & $2.6 \%$ & $97.4 \%$ \\
\hline 800 GeV Collider (1985) & 13.8 & 744 & 10 & $1.9 \%$ & $98.1 \%$ \\
\hline 800 GeV Fixed Target (1985) & 140.4 & 5,447 & 219 & $2.6 \%$ & $97.4 \%$ \\
\hline 800 GeV Fixed Target (1984) & 134.6 & 3,023 & 140 & $4.5 \%$ & $95.5 \%$ \\
\hline 400 GeV Fixed Target (1983/4) & 117.9 & 3,433 & 219 & $3.4 \%$ & $96.6 \%$ \\
\hline
\end{tabular}

It is encouraging that the current Collider Run II has not experienced higher satellite refrigerator downtime. Operation of the Tevatron at $980 \mathrm{GeV}$ required the installation of twenty-four cold helium compressors. The two-phase circuit of the magnet strings now operates subatmospheric. To date, operating in this mode has not adversely affected downtime.

TABLE 4 gives the downtime associated with quenches in the Tevatron. It is surprising that fixed target physics did not consistently register more downtime than colliding beam physics. With the constant beam manipulation associated with fixed target operation, a higher number of quenches is realized in fixed target physics. Having a similar amount of downtime for quenching in collider and fixed target modes implies that a longer recovery time is experienced in collider mode. This may be due to some level of shot setup, or preparation for shot setup, being charged to the quench recovery. In the $980 \mathrm{GeV}$ collider operation, longer quench recoveries are expected in order to restore the magnet string to the lower temperature required to operate at higher energy.

\section{CRYOGENIC UPGRADES}

Over the last decade, the Cryogenics Department has undertaken several major upgrades to the Tevatron cryogenic system. These upgrades were initiated to address one of three areas: to achieve the higher energy physics requirements for the current Collider Run II, to maintain a high level of reliability and redundancy in the cryogenic system and to improve the operational efficiency of the cryogenic system. A list of the major upgrades is presented below.

Central Helium Liquefier Cold Box II Construction

Completed: 1991

Purpose: To provide CHL redundancy and added capacity.

Method: Built with spare Cold Box I heat exchangers and with larger variable nozzle turbines.

Capacity: 5400 liters/hour 
Central Helium Liquefier Compressor D Construction

Completed: 1995

Purpose: To provide compressor redundancy during high capacity operation.

Tevatron Higher Energy Upgrade [7]

Completed: 1993

Purpose: Higher beam energy operation in the Tevatron.

Method: Lower the operating temperature of the Tevatron magnets using cold vapor compressors.

Results: $\quad 9 \%$ increase in accelerator beam energy in collider physics.

Tevatron Mycom Screw Compressor Efficiency Upgrade [10]

Completed: 1997

Purpose: Operating efficiency improvement.

Method: Higher efficiency screw profile and lower viscosity oil.

Results: $\quad 12.5 \%$ increase in isothermal efficiency.

Central Helium Liquefier Cold Box I Capacity Upgrade

Completed: 2001

Purpose: Regains cold box redundancy during higher energy operation.

Results: $\quad 20 \%$ increase in liquefier production capacity.

Central Helium Liquefier Higher Capacity Upgrade [11]

Completed: 2000

Purpose: Improved operating efficiency and capacity increase for Run II.

Method: Higher pressure cycle operation.

Results: $\quad 6400$ liters/hour peak capacity of each cold box.

Central Helium Liquefier Compressor C Restaging [11]

Completed: 2001

Purpose: Improved operating efficiency by allowing for better compressor matching to loads during Tevatron high beam energy operation.

Method: Reconfigured piping and changed cylinder sizes to achieve $33 \%$ higher flow rate using four stages (versus three stages).

\section{CONCLUSIONS}

The Tevatron proved that a large scale superconducting project could be built and reliably operated over a long period of time. Cryogenic upgrades were required to meet the growing demands on capacity and to ensure continued reliable operation. Operating for long periods of time have made the economics of efficiency improvement projects more favorable.

After twenty years of operation, the Tevatron continues to be the highest energy particle accelerator in the world. This will continue to be the case until the LHC becomes operational later in this decade. Long range scheduling shows Tevatron operations will continue for at least another ten years. As the Tevatron goes into its third decade of operation, attention will need to be given to aging infrastructure.

The technical and economic justifications for large scale superconducting projects have resulted in several other operational systems, with more in the design or construction phase. With each project, the cryogenic system designers and manufacturers are better able to ensure that an efficient, reliable and flexible system is realized. Continued cooperation between laboratories, universities and industry will be necessary to maintain the proper long-term knowledge base at all levels. 


\section{ACKNOWLEDGMENTS}

The author is grateful to all those who have contributed to the design, construction, operation and upgrade of the Tevatron cryogenic system. The success of the Tevatron physics runs over the last twenty years has required the insight, perseverance and dedication of many talented people. Fermilab is operated by Universities Research Association Inc. under Contract No. DE-AC02-76CH03000 with the United States Department of Energy.

\section{REFERENCES}

1. Theilacker, J. C., et al., "Commissioning of the Tevatron Satellite Refrigeration System," in Advances in Cryogenic Engineering 29, edited by R. W. Fast, Plenum Press, New York, 1984, pp. 437-444.

2. Theilacker, J. C., "Current Operating Experience and Upgrade Plans of the Tevatron Cryogenic System," in Advances in Cryogenic Engineering 35, edited by R. W. Fast, Plenum Press, New York, 1990, pp. 917923.

3. Peterson, T. J., "Fermilab's Satellite Refrigerator Expansion Engines," in Advances in Cryogenic Engineering 29, edited by R. W. Fast, Plenum Press, New York, 1982.

4. Rode, C. H., et al., "Fermilab Tevatron Transfer Line," in Advances in Cryogenic Engineering 27, edited by R. W. Fast, Plenum Press, New York, 1982, pp. 769-775.

5. Theilacker, J. C. and Rode, C. H., "An Investigation Into Flow Regimes for Two-Phase Helium Flow," in Advances in Cryogenic Engineering 33, edited by R. W. Fast, Plenum Press, New York, 1988, pp. 391398.

6. Klebaner, A. L. and Theilacker, J. C., "Thermal Performance of the Tevatron Magnets Under Higher Energy Operating Conditions," in Advances in Cryogenic Engineering 47, edited by S. Breon et al., American Institute of Physics, New York, 2002, pp. 84-89.

7. Theilacker, J. C., "Upgrade of the Tevatron Cryogenic System," in Advances in Cryogenic Engineering 39, edited by P. Kittel, Plenum Press, New York, 1994, pp. 517-522.

8. Theilacker, J. C., Cryogenics 34, pp. 107-110 (1994).

9. Walker, R. J., "Spectrographic Nitrogen Detector," Fermilab-TM-0742, 1977.

10. Martinez, A. and Pallaver, C. B., "Test of an Improved Oil Injected Helium Screw Compressor at Fermilab," in Advances in Cryogenic Engineering 47, edited by S. Breon et al., American Institute of Physics, New York, 2002, pp. 965-971.

11. Geynisman, M. G. and Makara, J. N., "Fermilab's Central Helium Liquefier Capacity Upgrade," in Advances in Cryogenic Engineering 47, edited by S. Breon et al., American Institute of Physics, New York, 2002, pp. 199-206. 